\title{
Aktuelle Probleme und Zukunftsperspektiven der ERG im Rahmen der Beziehungen Schweiz - Dritte Welt
}

„Round-table"-Diskussion zwischen Vertretern des Bundes, des Vororts, des SBV, des SGB und Hilfswerken, vom 10. Juli 1985 in Bern unter der Leitung des IUED, Genf

\section{OpenEdition}

\section{Journals}

Electronic version

URL: http://journals.openedition.org/sjep/1038

DOI: 10.4000/sjep.1038

ISSN: 1663-9677

\section{Publisher}

Institut de hautes études internationales et du développement

Printed version

Date of publication: 1 janvier 1985

Number of pages: 123-153

ISSN: 1660-5926

\section{Electronic reference}

"Aktuelle Probleme und Zukunftsperspektiven der ERG im Rahmen der Beziehungen Schweiz Dritte Welt », Schweizerisches Jahrbuch für Entwicklungspolitik [Online], 5 | 1985, Online erschienen am: 16 Februar 2013, abgerufen am 08 September 2020. URL : http://journals.openedition.org/sjep/1038 DOI : https://doi.org/10.4000/sjep.1038 
AKTUELLE PROBLEME UND ZUKUNFTSPERSPEKTIVEN DER ERG IM RAHMEN DER BEZIEHUNGEN SCHWEIZ - DRITTE WELT

"Round-table"-Diskussion zwischen Vertretern des Bundes, des Vororts, des SBV, des SGB und Hilfswerken, vom 10. Juli 1985 in Bern unter der Leitung des IUED, Genf 


\section{Einleitung}

Die Exportrisikogarantie, das wichtigste Exportförderungsinstrument des Bundes, ist aus zwei Gründen zum Thema wirtschafts- und entwicklungspolitischer Diskussionen geworden : ihre Eigenwirtschaftlichkeit ist gegenwärtig nicht gesichert und ihre Zielkonformität mit der schweizerischen Entwicklungspolitik wird in Frage gestelle.

Die ERG verzeichnet seit 1978 jährlich höhere Ausgaben als Einnahmen. Der in den Jahren zuvor angehäufte Reservefonds vermochte ab 1982 die Ausgabenüberschüsse nicht mehr zu decken. Seither leistet der Bund rückzahlbare Vorschüsse zur Deckung des Defizits, welche Ende Juni 1985623 Mio Fr. erreicht haben.

In den 70er Jahren wies die ERG nicht nur ein starkes Wachstum auf (1970: 3,38 Mia Fr. Gesamtverpflichtungen, 1979 : 25,4 Mia Fr., 1984 : 15,8 Mia Fr.) sondern sie verzeichnete auch seit Mitte der 70er Jahre einen steigenden Anteil der Lieferungen in die Entwicklungsländer (1975: $52 \%$, $1980: 57 \%$, $1984: 78,7 \%)$. Innerhalb der versicherten Lieferungen nach Entwicklungsländern wuchs zudem der Anteil der Lieferungen nach ärmeren Entwicklungsländern.

Nachdem seit 1981 laut dem ERG-Gesetz entwicklungspolitische Kriterien für Exporte nach den ärmeren Entwicklungsländern mitberücksichtigt werden müssen, erfolgte im Frühjahr 1985 eine Erhöhung der Gebühren und eine Sistierung der Absicherung von Währungsrisiken.

Im Frühling 1985 wünscht eine Einfache Anfrage Blunschy (CVP/SZ), den Kreis der ärmeren Entwicklungsländer zu erweitern, einzelne Evaluationen durchzuführen, die Erfahrung der Hilfswerke im Entscheidungsprozess zu nutzen und die Transparenz der Versicherung zu erweitern.

Eine in der Sommersession des Parlaments eingereichte Motion Renschler $(\mathrm{SP} / \mathrm{ZH})$ fordert eine unverzügliche Revision der ERG, um sowohl einer weiteren Belastung des Bundeshaushalts durch ERG-Defizite vorzubeugen, als auch eine kohärente Entwicklungs- und Aussenwirtschaftspolitik zu gewährleisten.

Bei diesem Stand der öffentlichen Diskussion hat die Redaktion sechs wichtige Vertreter des unterschiedlichen Meinungsspektrums zur ERG zu einer "Round-table"-Diskussion eingeladen. Diese fand am 10. Juli 1985 in Bern statt und ist im nachfolgenden Artikel dokumentiert. 


\section{Gesprächsteilnehmer :}

Dr. Richard Gester, Arbeitsgemeinschaft Swissaid/Fastenopfer/Brot für Brüder/Helvetas, Bern.

Dr. Peter Hutzli, Vorort des Schweiz. Handels- und Industrievereins, Mitglied der Kommission für die Exportrisikogarantie, Zürich.

Beat Kappeler, Schweiz. Gewerkschaftsbund, Bern.

Dr. Walter H. Rambousek, Generaldirektion Schweiz. Bankverein, Basel.

Dr. Peter Saladin, Bundesamt für Aussenwirtschaft, Präsident der Kommission für die Exportrisikogarantie, Bern.

Hans Schöb, Generaldirektion Schweiz. Bankverein, Basel.

\section{Redaktion Jahrbuch Schweiz - Dritte Welt, IUED}

Prof. Dr. Jacques Forster, Institut universitaire d'études du développement, Genf.

Hilmar Stetter, Journalist, Bern.

IUED - In der ersten Gesprächsrunde ist jeder Teilnehmer gebeten, aus seiner Sicht die Rolle der ERG im Rahmen der heutigen Aussenwirtschafts- und Entwicklungspolitik der Schweiz zu skizzieren, und die Hauptfragen, welchen sich seiner Meinung nach dieses Instrument schweizerischer Wirtschaftspolitik gegenübergestellt sieht, zu nennen.

\section{Die ERG ist grundsätzlich zweckmässig}

Saladin - Wir können mit der Feststellung beginnen, dass das wirtschaftliche Wohlergehen der Schweiz und ihrer Bevölkerung zu einem grossen Teil vom Export von Gütern und Dienstleistungen abhängt. In dieser Perspektive sind Erhaltung und Förderung der Wettbewerbsfähigkeit der schweizerischen Wirtschaft, insbesondere der aussenwirtschaftlich orientierten Zweige, ein prioritäres Erfordernis. Diese Wettbewerbsfähigkeit stellt einen unbestrittenen Beitrag an das wirtschaftliche, soziale und politische Ueberleben der Schweiz dar. Die Wettbewerbsfähigkeit ist ein komplexes Phänomen, das nicht leicht zu fassen ist. Die schweizerische Wirtschaft hat sich in der Vergangenheit als konkurrenzfähig erwiesen und hat deshalb diesen Beitrag an unser volkswirtschaftliches Wohl leisten können.

Der Bund verfügt für die Förderung der Wettbewerbsfähigkeit der schweizerischen Wirtschaft über verschiedene Instrumente. Das wichtigste besteht darin, dass er die allgemeinen Rahmenbedingungen für den Exporteur möglichst günstig gestaltet. Im engeren Bereich der Exportförderung stellt die ERG das zentrale Instrument des Bundes dar. Und es ist das zentrale Instru- 
ment für die Exporte in jene Länder, in denen das politische Risiko - um einmal diesen generellen Begriff zu gebrauchen - relativ hoch ist, und wo der Exporteur sich auf eine Garantie abstützen muss.

Ihre generelle Bedeutung muss im Rahmen unserer gesamten Exporte gesehen werden. Welcher Teil der jährlichen Exporte effektiv versichert wird, ist nicht einfach zu ermitteln, weil sich die Garantiegewährungen und die Exporte nicht decken, sondern jahreweise überlappen. Die durch die ERG abgestützten Exporte dürften in der Grössenordnung von ca. $5 \%$ unserer Gesamtexporte liegen (1984). Bezieht man den Anteil auf einzelne Länder, so sieht die Relation anders aus. Der grösste Teil der schweizerischen Exporte wickelt sich ja mit den OECD-Staaten ab - ca. $76 \%$. Diese Exporte laufen praktisch über die normalen Handelskanäle, benötigen also die ERG nicht. Meine Feststellung also : Die ERG ist ein zentrales Instrument für die Exportförderung in risikobehaftete Länder.

Hauptproblem : Dies ist sicher die finanzielle Situation der ERG. Wir können auf eine sehr erfolgreiche Versicherung zurückschauen, die von 1934 bis 1981 mit positivem Zahlen abgeschlossen hat und erst in den letzten drei bis vier Jahren effektiv vom gesetzlich verankerten Prinzip der Selbsttragung, wie es 1981 eingeführt wurde, zeitweise abwich, indem der Bund Vorschüsse leisten musste, die im übrigen zu verzinsen und auch zurückzubezahlen sind. Die Herstellung dieses finanziellen Gleichgewichtes ist sicher die Hauptaufgabe für die ERG in den nächsten Jahren. Im übrigen ist das System gesund, funktioniert zweckmässig und bedarf meiner Ansicht nach keiner grundsätzlichen Aenderungen.

\section{Die Berücksichtigung entwickluntspolitischer Kriterien bereitet der ERG keine Probleme}

Hutzli - Die ERG ist unbestrittenermassen ein Instrument zur Erhaltung und Schaffung von Arbeitsplätzen durch Förderung der Exporte. Diese Zielsetzung lag schon dem ersten Gesetz von 1934 zugrunde und ist nach wie vor gültig.

Der Zweckartikel wurde vor fünf Jahren durch die Bestimmung ergänzt, dass die ERG die Belange der schweizerischen Entwicklungspolitik mitzuberücksichtigen habe. Die ERG ist damit aber nicht ein Instrument der Entwicklungspolitik geworden. Sie ist nach wie vor eindeutig ein Instrument der Exportförderung. Die staatlichen Mittel zur Exportförderung sind in der Schweiz ohnehin recht bescheiden. Die Privatwirtschaft wünscht dies in der Regel auch nicht anders. Es wird aber immer wieder betont, dass die ERG die bedeutendste Exportförderungsmöglichkeit sei, die der Staat der Wirtschaft bietet.

Wir haben nun festgestellt, dass von seiten der Entwicklungshilfeorganisationen in letzter Zeit die ERG zunehmend unter Beschuss gekommen ist. Dabei werden zwei Dinge verknüpft. Einerseits wird geltend gemacht, es würden 
gewisse Aspekte der Entwicklungspolitik nicht genügend berücksichtigt; daneben wird jeweils auch die finanzielle Situation der ERG genannt, obschon dies zwei vollständig verschiedene Gebiete sind.

Das eine Problem liegt darin, wie die ERG finanziell wieder ins Lot gebracht werden kann; das ist aber kein Problem der Entwicklungspolitik. Das andere Problem ist die Frage, ob dem Grundsatz, die Aspekte der Entwicklungspolitik seien mitzuberücksichtigen, nachgelebt wird. Nach unserer Auffassung besteht hier aber überhaupt kein Problem.

Die Bedeutung der ERG für die ärmeren Entwicklungsländer darf nicht überschätzt werden. Es werden wenig Garantien für diese Gebiete gewährt. Das Problem wird nach unserer Auffassung von den Entwicklungshilfeorganisationen aufgebauscht. Angesichts gewisser Fehlschläge und der allgemein sich verbreitenden Ernüchterung über die Zweckmässigkeit der Entwicklungshilfe besteht von seiten der Entwicklungshilfeorganisationen nun die Tendenz, durch die Angriffe auf die ERG ihre eigenen Probleme zu vernebeln.

\section{Die Entscheidungsprozesse sollen nicht kompliziert werden}

Schoeb - Aus der Sicht der Banken möchte ich den Standpunkt wie folgt zusammenfassen : Im Rahmen der heutigen Aussenwirtschafts- und Entwicklungspolitik der Schweiz betrachten es die Banken als ihre wichtigste Aufgabe, die Exportindustrie mittels attraktiver Finanzierungen zu unterstützen. Für die Banken spielen dabei die Bedingungen der ERG eine entscheidende Rolle. Das Ziel der Banken ist somit im Rahmen der von den politischen Behörden erlassenen Vorschriften und Richtlinien, der Schweizer Exportwirtschaft die besten Dienstleistungen anzubieten. Grundsätzlich wollen und können die Banken keine Politik machen.

Die Stärken der Schweiz sind sicher die Qualität, die Zuverlässigkeit, die technisch hochstehenden Produkte. Diese Stärken versuchen die Banken zu kombinieren mit einer Finanzierung, die die gleichen Qualitäten hat. Wir glauben, dass die Schweiz im Aussenhandel sehr erfolgreich agiert, wenn die freien Marktkräfte zum Tragen kommen. Probleme sehen wir am ehesten dann, wenn das Preis-Leistungsgefüge durch ausländische Subventionen oder andere staatliche Unterstützungen verzerrt wird und die berühmten Schweizer Spiesse nicht so lang sind, wie diejenigen der Konkurrenz aufgrund der Subventionspolitik im Ausland.

Aus der Sicht der Banken sollte die Rolle der ERG auf Leistungen beschränkt sein, die der freie Markt nicht anbietet, die im Ausland aber bereitgestellt werden. Die Frage ist somit die, ob die Leistungen der ERG beibehalten oder ausgebaut werden sollen. Wir teilen die Sorge unserer Exportindustrie in diesem Bereich. Wir sehen einerseits, dass die ERG ihre Leistungen nicht ausbauen kann, ohne dass daraus erhöhte Defizite folgen. Andererseits haben wir grosse Bedenken, wenn sich die Schweiz aus innenpolitischen Ueberlegungen zusätzliche Erschwernisse aufladet in Form von freiwilligen Einschränkun- 
gen, wenn gleichzeitig im Ausland die Garantiegewährung mit wenig politischen Skrupeln angepackt wird. Das Schweizer System zeichnet sich durch seine Effizienz, seine einfachen Strukturen und seine Anpassungsfähigkeit aus. Das gilt vor allem für die Exportindustrie, es gilt auch für die ERG, und die Banken geben sich alle Mühe flexibel, schnell, einfach und anpassungsfähig zu sein. Wir haben grosse Bedenken, wenn die Entscheidungsprozesse beim Bund verlangsamt und relativ kompliziert werden sollen. Es berührt unangenehm, wenn im Zusammenhang mit Mischkrediten im Ausland mit wenigen oder keinen Vorbehalten Exportförderung betrieben wird und nicht Entwicklungshilfe.

Ich habe keine Schwierigkeiten, zur ERG-Politik meines Landes zu stehen. Den Preis für die weisse Weste, die sich die Schweiz bei der OECD holt, zahlen aber nicht die Politiker, sondern die Exportindustrie. Die Banken sind seit je bestrebt, die Stärken des Werkplatzes Schweiz mit denjenigen des Finanzplatzes Schweiz zu kombinieren und der ausländischen Konkurrenz aufgrund marktwirtschaftlicher Bedingungen die Stirne zu bieten. Rückschläge wurden in der Vergangenheit vor allem dann erlitten, wenn aufgrund politischer Unterstützung im Ausland die Schweizer mit deutlich kürzeren Spiessen in dem Kampf zogen. Diese Spiesse nochmals aufgrund innenpolitischer Ueberlegungen zu kürzen, scheint mir heute weniger verantwortbar denn je.

\section{Mehr Transparenz und Alternativen bei staatlichen Eingriffen}

Gerster - Ich möchte das Grundanliegen in Erinnerung rufen, weshalb die privaten Entwicklungsorganisationen sich mit der ERG befassen. Es geht uns um eine kohärente Entwicklungs- und Aussenwirtschaftspolitik, um die Vermeidung von Widersprüchen in den Zielsetzungen. Auch aus unserer Sicht kann es sich nicht um eine Umwandlung der ERG in ein Instrument der Entwicklungshilfe handeln. Denn die klare Trennung von Entwicklungsförderung und Exportförderung ist auch von uns anerkannt. Aber wir meinen, dass in den Beziehungen zu den Entwicklungsländern Widersprüchlichkeiten ausgeschaltet werden sollten.

Ich möchte aus meiner Sicht, der sich seit 1978 mit der ERG aus dem Blickwinkel der Entwicklungsorganisationen befasst, einige Stationen der Diskussion aufzeichnen. Ich beginne mit der Gesetzesänderung von 1981, die damals mit dem Hauptargument bekämpft worden ist, dass sie unnötig sei, weil man entwicklungspolitischen Anliegen ohnehin schon Rechnung trage. Damals wurde auch von Exponenten der Exportindustrie darauf hingewiesen, dass im Prinzip gar keine Rechtsgrundlage bestehen würde zur Ablehnung von Projekten aus entwicklungspolitischen Gründen, wenn nicht Risiko-Erwägungen usw. angeführt werden können. So beschloss dann das Parlament, dass bei Exporten in die ärmeren Entwicklungsländer künftig die Grundsätze der schweizerischen Entwicklungspolitik mitzuberücksichtigen sind. 
Wir waren damals der Meinung, dass damit das Ende der Auseinandersetzung gekommen sei. Seither hat sich dies aber erst als Beginn der Auseinandersetzung herausgestellt, weil es nämlich seit dieser Gesetzesänderung darum ging, diese auch in die Praxis überzuführen. Und wir kamen nicht um den Eindruck herum, dass diese Klausel anfänglich als lästige Zusatzbestimmung ignoriert wurde. Dies ging beispielsweise auf seiten der Industrie aus einer Broschüre des VSM hervor, wo die entwicklungspolitische Zusatzbestimmung überhaupt nicht erwähnt worden ist. Ich finde eine gewisse Bestätigung im Votum von Herrn Hutzli heute, wenn er sagt, dass er in dieser Richtung überhaupt keine Probleme sehe.

Wenn ich gesagt habe, die Auseinandersetzungen haben 1980 erst richtig begonnen, möchte ich das am Begriff der ärmeren Entwicklungsländer illustrieren. Er ist ja als Kompromissformel im Gesetz verankert worden, nachdem der Nationalrat ursprünglich die Anwendung entwicklungspolitischer Grundsätze gegenüber allen Entwicklungsländer beschlossen hatte. Wir haben uns 1981 mit der Frage an das BAWI gewandt, gegenüber welchen Ländern diese Zusatzbestimmung nun angewandt würde ? Es wurde zuerst erwähnt, dass dies gegenüber den ärmsten Entwicklungsländern gemäss UNO-Definition der Fall sei. Dann haben wir auf eine schriftliche Anfrage hin den Bescheid erhalten, dass es gar keine fixe Länderliste gäbe. Auf einen parlamentarischen Vorstoss hin hat dann der Bundesrat bekannt gegeben, dass die Liste der einkommensschwächeren Entwicklungsländer gemäss OECD mit einem durchschnittlichen Pro-Kopf-Einkommen von 600 Dollar massgebend sei. Sie wissen, dass wir heute eine Gleichbehandlung des Begriffs der ärmeren Entwicklungsländer im Rahmen der Entwicklungszusammenarbeit wie auch der ERG wünschen, indem man die Obergrenze bei 1400 Dollar ansiedelt.

Ich könnte weitere einzelne Elemente darstellen, in denen klar wird, dass jede Umsetzung erkämpft werden muss. Und ich meine, dass es doch ein aussergewöhnlicher Umstand ist, wenn man um die Anwendung eines gesetzlichen Erlasses kämpfen muss.

Zum Schluss ist es mir ein Anliegen, auf die Situation in Afrika hinzuweisen. Wenn Sie den Weltbankbericht des letzten Jahres zur Situation in Afrika durchsehen und das Kapitel über die Wurzeln des Problems aufschlagen, dann sehen Sie an prominenter Stelle die Begründung, dass die Verbreitung nichtlebensfähiger Grossprojekte in Afrika die Krise substantiell gefördert hat. Die Weltbank weist selber auf die Mitverantwortung - nicht auf die Alleinverantwortung - der Finanzgeber hin. Nachdem ja in Afrika die Exportkredite eine bedeutende Rolle spielen, sind - im Gegensatz zu den anderen Kontinenten - insbesondere die Exportkreditagenturen von dieser Mitverantwortung angesprochen. Sie wissen besser als ich, dass heute in der OECD und in der Weltbank Gespräche stattfinden, wie die Vergabe von Exportkrediten auf die entwicklungspolitischen Prioritäten ausgerichtet werden kann. Man musste nämlich feststellen, dass sehr viele Grossprojekte immer wieder im Widerspruch standen zur Entwicklungsstrategie, die in diesen Ländern verfolgt worden ist. 
In diesem Sinne, würde ich meinen, sind die entwicklungspolitischen Anliegen an die ERG aktueller denn je. Sie haben zudem eine internationale Dimension erhalten, mit der wir ursprünglich nicht gerechnet haben.

\section{Es geht um Kohärenz in der Aussenwirtschafts- und Entwicklungspolitik}

Kappeler - Die Exportrisikogarantie ist ein Instrument der Handelsförderung in der Schweiz. Sie zielt auf die Sicherung der Lage der einzelnen Unternehmen ab, welche sich dank einem gemeinsamen Versicherungspool unter staatlicher Garantie rückversichern und damit ihr Ueberleben sichern können. Arbeitsplätze sind erst indirekt im Spiel, denn ich würde die Förderung des Handels und der Unternehmungsstabilitäten als das Hauptziel dieser Versicherung bezeichnen. Die Erhaltung der Arbeitsplätze ist allenfalls ein Resultat, ein unsicheres zudem, das von verschiedenen Faktoren abhängt, wie beispielsweise der wachsenden Kapitalintensität und der Technisierung. Diese sind im Exportsektor höher als in allen binnenwirtschaftlichen Sektoren, mit Ausnahme der Elektrizitätsindustrie und der Zementindustrie. Dies muss vorausgeschickt werden, wenn direkt mit Arbeitsplätzen argumentiert wird.

Damit est angedeutet, dass möglicherweise mit gleichem staatlichen Mitteleinsatz, mit einer halben Milliarde Defizitgarantie, wie sie jetzt aufgelaufen ist, im Binnensektor sogar mehr Arbeitsplätze geschaffen werden könnten. Allerdings : wenn keine Garantie bestünde, beispielsweise auf privater Ebene, hätten einzelne Unternehmungen die Segel streichen müssen, und das wäre sicher zulasten der betroffenen Arbeitsplätze gegangen.

Die Einrichtung der ERG darf aber, obwohl sie ein Instrument der Handelsförderung ist, also ein egoistisches Instrument, der Idee und der Zielsetzung der Entwicklungszusammenarbeit der Schweiz nicht zuwiderlaufen. Von daher unterstützen wir das seinerzeit angenommene Amendment und finden, dass, wenn der Staat einerseits eine Entwicklungspolitik mit Hunderten von Millionen Franken Einsatz führt und auf der anderen Seite eine Handelsförderungspolitik mit ebenfalls Hunderten von Millionen Franken Einsatz, sich diese zwei Politiken nicht beissen sollten. Und ein Interessenkonflikt sollte so gelöst werden, dass sicher nicht die Aermsten der Welt die Leidtragenden sind.

Die ERG ist ein grosser staatlicher Eingriff, sie ist nicht strukturneutral, in dem Sinn, als der Staat bestehende Firmen damit stützt. Andere Eingriffe, die in der schweizerischen Politik zur Zeit diskutiert werden, wie beispielsweise die Innovationsrisikogarantie, sind in dem Sinn auch nicht strukturneutral; sie berücksichtigt Neuunternehmungen, Kleinunternehmungen und technologisch neue Unternehmungen, die ERG begünstigt hingegen grosse Unternehmen, bestehende Unternehmungen. Ich sage nicht, dass sie Kleinen nicht auch nützt, aber sie begünstigt eher die Grossen, weil die in diesem Geschäft tätig sind. Sie stützt auch bestehende Techniken und vor allem Grosstechniken, währenddem andere Instrumente wie eben die Innovationsrisikogarantie 
neue Techniken begünstigen würden. Die ERG greift in die Strukturen ein. Sie erhält, und das ist auch ein Eingriff.

Die Bundesfinanzen sind von der ERG recht stark betroffen in Zeiten, da die ERG sich nicht selber deckt. Es ist fraglich, ob das aufgelaufene Defizit durch die Beiträge der versicherten und interessierten Industrie gedeckt werden kann, und von daher besetzt die ERG einen gewissen Platz in der gesamten Finanzpolitik. Deshalb muss der Mitteleinsatz, der hier durch den Staat geleistet wird, verglichen werden mit den "opportunity-costs", also mit den anderen Möglichkeiten, die diese Summen und ihr Einsatz auf Arbeitsplätze, auf Wirtschaftspolitik und Wirtschaftsstruktur und auf die Entwicklungspolitik haben könnten. Die ERG muss sich diesen Vergleich gefallen lassen, solange sie durch den Bund in dieser Höhe finanziert wird.

Und schliesslich : die Transparenz. Aufgrund des strukturpolitischen Interesses, des entwicklungspolitischen Interesses und des finanzpolitischen Interesses wird verlangt, dass Transparenz hergestellt wird, wie in jedem anderen Sektor öffentlicher Tätigkeit. Diese Transparenz ist gegenwärtig in absolut ungenügender Weise realisiert. Sie wäre durch Beteiligung weiterer interessierter Kreise an der Durchführung und der Verwaltung der ERG herzustellen.

Rambousek - Ich möchte aus meiner Sicht vor allem auf einen Punkt eingehen und ihnen einige Daten über die Verteilung der Schweizer Exporte präsentieren, die uns im weiteren Verlauf der Diskussion hilfreich sein können. Der Anteil der OECD-Länder am Total der Schweizer Exporte hat von 1975 bis 1984 von 71 auf $76 \%$ zugenommen. Die verschiedenen Entwicklungsländergruppen sind jeweils mit wenigen Prozentanteilen am Total der Schweizer Exporte beteiligt, und zwar auch retrospektiv bis 1975 betrachtet.

Wenn wir die 67 ärmeren Entwicklungsländer nehmen, das wären also jene Länder, für die im Rahmen der ERG einige entwicklungspolitische Vorsicht am Platz sein muss, dann stellen wir fest, dass deren Anteil an den Schweizer Exporten von 1975 bis 1984 von 2,9 auf 3,2\% zugenommen hat, also immerhin um $10 \%$, aber von einer sehr niedrigen Basis aus. Für die 31 ärmsten Länder nach der OECD-Klassifikation ergibt sich der sehr, sehr kleine Anteil von 0,5 beziehungsweise von $0,4 \%$.

Wenn wir in die mittlere Entwicklungsländergruppe gehen, also jene Länder nehmen, die ein Pro-Kopf-Einkommen von über 600 Dollar haben, so stellen wir eine Verminderung von 6,4 auf 5,5\% fest. Bei der Gruppe der elf Schwellenländer nehmen wir ebenfalls eine Abnahme wahr : von 11,5 auf 8,6\%. Die 11 OPEC-Länder, ohne Nigeria und Indonesion, verzeichnen eine leichte Zunahme.

Uns interessieren ja kaum der OECD-Anteil, sondern die 67 ärmeren Länder, die mittleren Entwicklungsländer und die Schwellenländer. Und wir stellen bei den mittleren Entwicklungsländern und den Schwellenländern, bei denen sich die Frage der Wettbewerbsfähigkeit entscheidend stellt, eine Ab- 
nahme dieses Anteils fest. Es stellt sich also die Frage, wie dieses Instrument auszugestalten ist, so dass die Wettbewerbsfähigkeit, die von Herrn Saladin am Anfang erwähnt wurde, eben weltweit für unsere Exportindustrie erhalten werden kann.

\section{Die Frage der Mitberücksichtigung entwicklungspolitischer Kriterien}

Saladin - Ich wehre mich gegen die Meinung von Herrn Gerster, die ERGOrgane wollten Art. 1, Abs. 2 nicht anwenden. Wir kennen den gesetzlichen Auftrag und haben uns seit der Inkraftsetzung dieses Artikels im Jahre 1981 bemüht, diesem Gesetzesauftrag zu entsprechen. Wenn am Anfang in bezug auf die Länderdefinition eine gewisse Unsicherheit bestand, so deshalb, weil man vorerst gewisse Erfahrungen sammeln musste, um zu sehen, was unter ERG-Gesichtspunkten der Begriff "ärmere Länder" überhaupt bedeuten kann. Aber schon Anfang 1982 hat der Bundesrat in Beantwortung einer Einfachen Anfrage die heute geltende Definition bekanntgegeben.

Der gesetzliche Auftrag. Herr Kappeler, ist ganz klar die Arbeitsbeschaffung und die Förderung des Aussenhandels. Artikel 1, Absatz 1 hält fest : “Der Bund kann im Interesse der Schaffung und Erhaltung von Arbeitsgelegenheiten und der Förderung des Aussenhandels die Uebernahme von Exportaufträgen, bei denen der Zahlungseingang mit besonderen Risiken verbunden ist, durch Gewährung einer Garantie erleichtern."

Die Transparenz : Wir haben uns bemüht, in den jährlichen Pressemitteilungen eine weitgehende Transparenz über die Operationen und die Resultate der ERG zu schaffen.

Kappeler - Ich habe Beteiligung am Mechanismus, an der Durchführung gefordert.

Gerster - Ich muss darauf hinweisen, dass es sich in keiner Weise um eine Unterschiebung handelt ! Vielmehr habe ich bereits am Begriff der ärmeren Länder belegt, dass es einen Stationenweg der Auseinandersetzungen gibt, wie sich im einzelnen die Umsetzung dieser Zusatzbestimmung in die Praxis vollzogen hat - soweit dies überhaupt von aussen wahrnehmbar sit. Und damit sind wir wiederum beim Problem der Transparenz.

Ich möchte nun aber anhand eines zweiten Beispiels den mühsamen Weg vom Gesetz zur Praxis erläutern. 1981 erfolgte die Anfrage Blunschy. Der Bundesrat teilte mit, dass die Informationsbeschaffung auf umfassende Art und Weise erfolge und unter anderem auch Informationen von den Unternehmen eingeholt würden, wenn ein entsprechendes Garantiegesuch vorliege. Als dann einige Zeit später das Gesuch der Firma Losinger für den Bau des Manantali-Staudamms zur Diskussion stand und ich mich bei der Firma erkundigte, hatte Losinger nicht einmal eine Ahnung davon, dass bei der ERG entwicklungspolitische Ueberlegungen mitzuberücksichtigen sind. Ich habe 
in Gesprächen mit Exporteuren wiederholt festgestellt, dass die Kenntnis dieser Zusatzbestimmung betreffend Exporte in ärmere Entwicklungsländer kaum vorhanden ist. Von daher gibt es offensichtlich zahlreiche Elemente, die auf die Meinung hindeuten, dass alles wie bisher weitergehe. Ich kann mir aufgrund öffentlicher Diskussionen und verschiedener Demarchen in letzter Zeit vorstellen, dass in der Praxis seitens der ERG-Komission diesen Aspekten nun vermehrt Rechnung getragen wird. So nehmen wir von aussen den Umgang mit dieser Zusatzbestimmung wahr.

Hutzli - Ich möchte auf das Votum von Herrn Kappeler zurückkommen. Er vermischt wieder einerseits die Entwicklungsaspekte, und andererseits die Rolle der ERG für die schweizerische Wirtschaft, ihre finanziellen Auswirkungen für den Bund und ihren Einfluss auf die Struktur der Wirtschaft. Das ist ein Problemkreis, der mit Entwicklungspolitik nicht zu tun hat. Das sind zwei getrennte Probleme. Und ich gehe davon aus, dass wir hier den Entwicklungshilfeaspekt behandeln.

Zu Herrn Gester : Wenn Sie sagen, Sie hätten im Jahre 1981 geglaubt, dass die Diskussion mit der Annahme des Blunschy-Amendments zu Ende sei, dann sind Sie nicht ehrlich. Sie sind mit Ihrem Antrag, den Sie über die Kommission Blunschy eingereicht haben, nur teilweise durchgedrungen. Der Antrag Renschler auf einen Sitz in der ERG-Kommission wurde deutlich abgelehnt. Sie wussten damals ganz genau, dass Sie die Diskussion weiterführen wollen. Sie sagten, Sie seien erst gezwungen worden, die Diskussion fortzuführen, als der Bund nicht entsprechend dem Amendment reagiert habe.

Bei der ERG-Kommission haben wir uns immer wieder damit beschäftigt, wie man diesem Amendment operationell am besten Rechnung trägt. Es ist ja auch eine Unterschiebung, zu behaupten, die ERG-Kommission sei über diese Gesetzesänderung hinweggeschritten.

$\mathrm{Zu}$ Losinger : Wir können natürlich nichts dafür, wenn nicht jede Firma dieses Gesetz liest. Aber die Hauptsache ist, dass es der ERG-Kommission bekannt ist.

\section{Welche Bedeutung hat die ERG für die Arbeitsplätze ?}

IUED - Wir möchten, auch wenn die Entwicklungspolitik im Vordergrund steht, auf die Bedeutung der ERG für die Arbeitsplätze in der Schweiz zurückkommen. Der Arbeitsmarkt und die Struktur der Schweizer Industrie befinden sich gegenwärtig in einer Phase des technologischen Strukturwandels und der Internationalisierung der Produktionsstandorte. Welche Rolle spielt die ERG heute, wenn es darum geht, die Arbeitsplätze in der Schweiz zu erhalten und zu fördern?

Kappeler - In der Tat ist das Arbeitsplatzargument ja immer das vordergründigste, das einem um die Ohren geschlagen wird, und das auch im Ge- 
setzestext festgehalten wird. Das ist quasi die Geiselnahme der Arbeiterschaft für Dinge, die etwas ganz anderes bezwecken, beispielsweise Handelsförderung und Sicherung der Unternehmungen.

Das ist ja doch das primäre Ziel. Und um das geht es immer, die Arbeitsplätze sind ein abgeleiteter Effekt. Dieser abgeleitete Effekt hängt von verschiedenen Faktoren ab, unter anderem immer mehr davon, ob die Exportindustriearbeitsintensiv produziert, ob sie zum Beispiel Teile dieser Aufträge, die sie erhält, selbst durchführt, oder ins Ausland vergibt. Solche Fälle sind gerade jetzt wieder passiert, so dass für mich das Arbeitsplatzargument nicht derart im Vordergrund steht, wie es der etwas euphorische Gesetzesartikel und auch die anderen Aeusserungen zur ERG jeweilen tun.

Strukturpolitisch werden die Arbeitsplätze in einzelnen Sektoren unter die Käseglocke der ERG-Finanzierung gestellt, z.B. wird der Grossmaschinenbau in der Elektrizitätsproduktion gestützt. Damit werden aber nicht jene Teile der Industrie gestützt, die vielleicht im Interesse der Unternehmungen und der Arbeitsplatzzahl neue, erfolgsversprechendere Sektoren werden könnten. Kleinkraftwerke z.B. können sicher leichter und mit entwicklungspolitisch weniger riskanten Auswirkungen abgesetzt werden.

Strukturpolitische Eingriffe zeitigen in jedem Fall Auswirkungen auf die Arbeitsplätze. In diesem Sinn sind die Arbeitsplatzeffekte nicht einfach garantiert und gegeben, sondern variieren, je nach dem, wie sich die Förderung durch die ERG im Einzelfall und als Summe der Einzelfälle in der Gesamttendenz langfristig auswirkt.

Hutzli - Zur Frage der Arbeitsplatzerhaltung : Es ist schwierig, in Zahlen auszudrücken, wieviele Arbeitsplätze dank der ERG erhalten werden. Der Anteil darf aber nicht überschätzt werden, weil der weitaus grösste Teil der Exporte ohne ERG abgewickelt wird. Wir sehen aber auch immer wieder, wie empfindlich in der Schweiz bereits auf eine geringe Arbeitslosenrate reagiert wird.

Wir sind, das ist auch wichtig, hier gar nicht autonom. Unsere Exportindustrie brauchte die ERG nicht, wenn sie das Ausland auch nicht hätte. Solange aber ausländische Firmen von ihrem Staat diese Möglichkeit erhalten, wären unsere Firmen ohne ERG mit kürzeren Spiessen ausgestattet. Die Exportfinanzierung ist ja zu einem immer wichtigeren Faktor geworden. Heute entscheiden neben Qualität und Preis immer häufiger die Kreditbedingungen, die der Exporteur anzubieten vermag, über den Erhalt des Auftrages.

Und daraus hat sich weitgehend der ganze Kreditwettlauf unter den Industriestaaten entwickelt, den man mit dem OECD-Konsensus von 1976 etwas zu steuern versucht, allerdings mit beschränktem Erfolg! Und wenn unsere Banken bereit sind, unserer Exportindustrie durch die Gewährung von Exportkrediten beizustehen, hat das natürlich seine Grenze dort, wo das Risiko auch für die Banken zu gross wird. Und neben dem grossen Kuchen der Exporte, die ohne grosse Risiken abgewickelt werden können, gibt es dieses unter- 
schiedlich breite Band derjenigen Exporte, die nur noch mit der ERG getätigt werden können. In diesen Fällen hat die ERG einen direkten Einfluss auf die Arbeitsplätze.

Hier ist noch etwas zu sagen : Auch wenn der Anteil von Beschäftigten im Verhältnis zum Exportpreis gesunken ist, also ein Angestellter einen höheren Wert exportiert als früher, darf man nicht darüber hinwegsehen, dass nicht nur gerade der Mann, der an dieser Maschine, die exportiert wird, arbeitet, betroffen ist. Heute ist die ganze Dienstleistungsgesellschaft, die auch vom sekundären Sektor abhängt, immer grösser geworden. Wenn ein Einbruch in der Exportindustrie stattfindet, hat das Auswirkungen auf das Handwerk, auf das Gewerbe. Das hat einen Schneeballeffekt. Deshalb darf man die Arbeitsplatzsituation nicht herabspielen.

Noch ein anderer Aspekt : das Währungsrisiko. Das ganze Problem mit der ERG begann ja nicht wegen der politischen Risiken, sondern wegen der Währungsrisiken in den Jahren 77, mit einer Kulmination 78 und 79. Und dort war die ERG das einzige Mittel, das dem Exporteur zur Verfügung gestellt werden konnte, um diese Währungsrisiken einigermassen bewältigen zu können. Der Bund war ja auch machtlos, und von Bundesratseite wurde immer wieder betont, die ERG werde grosszügig eingesetzt. Das wurde dann sehr schnell vergessen, als die Lage sich etwas normalisierte, und dann die Rechnung aufgrund der Verluste präsentiert wurde.

Kappeler - Ich würde aus der Beobachtung kürzlicher grosser ERG-Fälle sagen, dass davon eher Firmen profitieren, deren Wertschöpfungsüberschuss eher gering ist und dass, meiner Ansicht nach, wenn schon Bundesgelder in dieser Höhe locker gemacht werden, andere Verwendungen ebenfalls diskutiert werden müssen. Hätte man für die halbe Milliarde, die man jetzt in die ERG gesteckt hat, die Nachrichtenindustrie gefördert, so dass sie z.B. in der Lage wäre, ein digitales Telefon-System anzubieten, dann hätten wir wahrscheinlich eine Exportindustrie mit sehr hohen Wertschöpfungen. Ich komme daher zwar nicht zum Schluss, die ERG sichere keine Arbeitsplätze, aber dass sie nicht einfach dieses Mannah ist, das gut für die Arbeitsplätze ist.

Schöb - Ich möchte noch einen Punkt betonen, der jetzt mehrmals gefallen ist. Der Fakturierung nach mag es wohl sein, dass hauptsächlich Grossunternehmungen in den Genuss der ERG kommen. Die Grossunternehmen bestätigen uns aber immer wieder, dass Dutzende von Unterlieferanten in der Schweiz direkt Arbeit bekommen, wenn sie einen Grossauftrag erhalten und die ERG in Anspruch nehmen.

Kappeler - Und im Ausland!

Schöb - Der Auslandanteil, der ERG-versichert werden kann, ist recht genau fixiert, ich glaube, jenen Teil können wir auf der Seite lassen. Ich rede von Unterlieferanten in der Schweiz und Arbeitsplätzen in der Schweiz ! 
In der Diskussion scheint mir manchmal, dass wir von Lieferungen in die ärmsten Entwicklungsländer reden, als ob wir damit die grosse Masse der ERG-versicherten Exporte meinten. Bei den Banken machen wir uns aber viel mehr Sorgen um die Exporte in die stärkeren Entwicklungs- und Schwellenländer, die nach der Statistik etwa $13 \%$ oder $14 \%$ aller Exporte der Schweiz ausmachen. Ob wir dort unsere Position halten können, ist entscheidend, und viel weniger die rund 4 oder fast 5-mal kleineren Exporte in die ärmsten Entwicklungsländer. In der Diskussion sollten wir diesem Gewicht Rechnung tragen. Die Sorge der Exportindustrie und der Banken gilt unserer Konkurrenzfähigkeit in den wichtigen Märkten.

Das Beispiel, das mit dem Manantali-Staudamm gegeben worden ist, ist eine doppelte Ausnahme, sowohl hinsichtlich Betrag wie Land.

Hutzli - Nur eine kurze Bemerkung zu Herrn Kappeler. Es ist sicher richtig, dass es auch andere Formen der Arbeitsplatzerhaltung gibt; bei der ERG tritt die Arbeitswirksamkeit vielfach aber bereits am Tage nach der Garantiegewährung ein. Bei anderen Projekten, die eine bestimmte Branche fördern wollen, ist dies vielleicht erst in 5 oder 10 Jahren oder überhaupt nie der Fall.

Saladin - Die Wettbewerbsfähigkeit und die erfolgreiche Strukturanpassung der schweizerischen Industrie hängt sicher nicht in erster Linie von der ERG ab. Diese entscheiden sich vor allem auf den Märkten der Industriestaaten, auf denen wir 3/4 unserer Exporte abwickeln, und für die, wie erwähnt, praktisch die ERG nicht beansprucht wird. Im übrigen steht der Exporteur fast immer in einer Konkurrenzsituation, und der Abnehmer entscheidet aufgrund von Preis, Qualität und finanziellen Bedingungen. Dabei est zu bedenken, dass der schweizerische Exporteur bei der Festlegung der finanziellen Bedingungen wenig Spielraum hat : denn die ERG hält sich an die im Rahmen des unter den OECD-Länder vereinbarten Konsensus, d.h. jeder andere Konkurrent kann im wesentlichen die gleichen finanziellen Bedingungen anbieten.

Es stehen ihm im Gegensatz zu den Konkurrenten aus den meisten anderen Industriestaaten keine staatlichen Subventionsinstrumente zur Verfügung, mit denen er seine Mitbewerber ausstechen könnte. Unser Mischkredit ist ja bekanntlich kein Mittel der Exportförderung, sondern der Entwicklungszusammenarbeit; bei seiner Abwicklung wird zudem, wo immer möglich, das Prinzip des internationalen Wettbewerbes verfolgt.

\section{Verfeinerte Beurteilungen schliessen Schäden nicht aus}

IUED - Seit den 70er Jahren pflegt die Schweiz einen verstärkten Aussenhandel mit einzelnen Regionen der Entwicklungs/änder. Gerade diese Regionen stecken nun in einer tiefen Wirtschaftskrise, und der stagnierende Export wird in einem Klima verschärfter Konkurrenz abgewickelt. Ich denke einerseits an die armen Entwicklungs/änder und zum andern Teil an die Schwel- 
lenländer, insbesondere die südamerikanischen Schwellenländer. Welche Bedeutung spielte die ERG bei dieser Markterschliessung und wie wurden die Länderrisiken eingeschätzt?

Schöb - Die Banken sind heute etwas einsichtiger für eine ganze Anzahl Entwicklungen als noch vor 10 Jahren. Wir würden sicher nicht alles wiederholen, was wir in den 70er Jahren in Form von Finanzierungen bereitgestellt haben. Wir haben eine rasche und starke Entwicklung vor allem in den Schwellenländern unterstützt, die schliesslich teilweise nicht durchgehalten werden konnte. Wir haben mit dem Rest der Welt die Auffassung geteilt, dass Brasilien, Argentinien, dass Oel fördernde Länder wie Mexiko tatsächlich eine wirtschaftliche Leistungsfähigkeit erreichen werden, die den Schuldendienst gewährleistet. Wir haben auch die Qualität der Projekte etwas höher eingestuft, als sie teilweise herausgekommen ist.

Heute haben diese Länder die bekannten Schwierigkeiten, ihre Schulden zu bedienen. Unsere Verantwortung ist die, diesen Ländern durch Anpassung der Kreditbedingungen Luft zu verschaffen, die sie zum Ueberlegen brauchen. Wir haben uns zur Einsicht durchgerungen, dass die Laufzeiten entsprechend lang, die "grace periods" grosszügig bemessen sein müssen, um das Ganze auf eine realistische Basis zu bringen. Wenn die Restrukturierungsbedingungen schliesslich doch nicht ganz so grosszügig ausfallen, so kommt der Widerstand selten von den Schweizer Banken.

Die Banken sind nicht nur gezwungen, die fälligen Beträge auf 10 und mehr Jahre zu restrukturieren. Weitere Verpflichtungen der Banken bestehen darin, im kurzfristigen Bereich durch Aufrechterhaltung der bestehenden Kreditlimiten den Import lebenswichtiger Güter sicherzustellen. Schliesslich leisten die Banken einen massgeblichen Beitrag zur Restrukturierung der Schulden in Form von Neugeld, das diesen Ländern zur Verfügung gestellt werden muss, bis sie wieder Zugang zum internationalen Kreditmarkt finden.

Zur Entwicklung der Exporte in den 70er Jahren : Ich glaube nicht, dass die Lieferkapazitäten der Schweiz ausgeschöpft worden sind. Es gab eine Anzahl grosser Aufträge, die aus politischen Ueberlegungen nicht in der Schweiz plaziert wurden, obwohl Schweizer Lieferanten hart darum gekämpft haben. Vom Produkt und der Finanzierung her hätte die Schweiz mithalten können. Aber weil im Ausland mit deutlich stärkerer staatlicher Unterstützung aufgefahren werden konnte, gingen die Aufträge für unser Land verloren.

Rambousek - Ich möchte zum Punkt der Beurteilung der Länderrisiken Stellung nehmen. Ich möchte darauf hinweisen, dass das systematische und verfeinerte Beobachten von Vorgängen wirtschaftlicher, politischer Natur über die ganze Welt, zur Zeit für 180 Länder, noch nicht so alt ist. Es war rein von der Datenlage her vor 10 Jahren viel schwieriger als heute, diese Länderbeobachtung durchzuführen. Diese ganzen Datensets werden von den internationalen Organisationen - jetzt auch vom IIF (Institute of Interna- 
tional Finance) in Washington, einer Forschungsinstitution der Banken, permanent verfeinert. Diese Länderrisikenbeurteilung ist sicher noch nicht abgeschlossen.

Hutzli - Ich habe schon erwähnt, dass in den siebziger Jahren die Bedeutung der ERG für die Währungsrisiken weit grösser war als für die politischen. Dort hatte sie eine wichtige Funktion, doch sind auf diesem Gebiet auch die grossen Verluste eingetreten. Die ERG spielte daneben teilweise sicher eine Rolle im Aufbau gewisser Märkte, so z.B. in den Oststaaten.

Die eigentlichen politischen Verluste, die aus dieser Zeit stammen, sind höchst gering. Die Politik, die die ERG damals verfolgt hat, kann man als vorsichtig und vernünftig bezeichnen. Es war auch nicht vorauszusehen, wie sich die einzelnen Regionen entwickeln würden. Länder, die vor 15 oder 20 Jahren gleiche Startbedingungen hatten, haben sich sehr unterschiedlich entwickelt, z.B. Afrika und Südostasien.

Ghana, zum Beispiel, ist im Grunde ja ein reiches Land, aber aus politischen und anderen Gründen konnte es aus dieser Gegebenheit kein Kapital schlagen. Anderen ist es gelungen, Singapur oder Taiwan z.B. Ein spezielles Problem ist natürlich Südamerika. Mexiko ist ein Oelförderland. In anderen Oelländern waren die Risiken auch gegeben und dort haben wir für die ERG praktisch keine Schäden zu verzeichnen. Wir sind ja sogar im Iran - wo noch politische Komponenten dazu kamen - mit einem sehr hellblauen Auge davon gekommen. Ich glaube sagen zu können, die ERG ist sehr vorsichtig gewesen und hat sich nicht kopflos auf diese neuen Märkte gestürzt. Erst die heutigen Konsolidierungen haben dann die politischen Schäden ansteigen lassen.

Saladin - Das Parlament hat ja die Aktivierung der Währungsrisikoversicherung als politischen Akt in einer schwierigen konjunkturellen Phase der Schweiz gefordert. Es war also nicht der Leichtsinn der ERG-Organe ! Das ERG-Loch rührt ganz eindeutig von der Währungsgarantie her. Es sind bis Ende 1984 für 1230 Millionen Franken Währungsgarantieverluste entstanden, und davon konnten etwa 600 Millionen - rund die Hälfte - durch Einnahmen abgedeckt werden; die andere Hälfte entspricht ungefähr den $600 \mathrm{Mil}$ lionen Franken, welche die ERG heute auf dem Buckel hat.

Bei der Länderrisikobeurteilung haben wir alle dazugelernt. Diese verfeinern wir auch innerhalb der ERG. Im BAWI haben wir ein Länderinformationsund Beurteilungssystem aufgebaut, das computergestützt ist und das es uns erlaubt, sowohl die kurz- wie die längerfristigen Entwicklungen in einem Land systematisch zu verfolgen. Aber auch damit lassen sich Schäden nicht vermeiden.

Kappeler - Zu den Währungsrisiken hat der Gewerkschaftsbund 1979 in der Vernehmlassung Vorschläge gemacht, die wahrscheinlich den allergrössten Teil der Verluste verhindert hätten. Wir schlugen damals vor, dass ein Gradualismus eingeführt wird. Je verschuldeter ein Land entsprechend gewisser Kri- 
terien ist (zum Beispiel Exporteinnahmen im Verhältnis zum Schuldendienst), um so weniger kann die ERG für Lieferungen in dieses Land beansprucht werden. Dieser Vorschlag wurde nicht aufgenommen, hätte aber wahrscheinlich sehr viel Geschirr ganz behalten - und ich wundere mich eigentlich, dass solche Vorschläge einfach so unter den Tisch geraten.

Dann möchte ich sagen, dass eine gewisse kritische Solidarität mit der Dritten Welt geübt werden muss. Kritisch in dem Sinn, als natürlich zugestanden werden muss, dass die Nachfrage nach Produkten nicht nur der raffinierten Verkaufspolitik von Konzernen zu verdanken ist, sondern dass tatsächlich eine bestehende Oberschicht in verschiedenen Dritte-Welt-Ländern eine Art von Entwicklungsweg eingeschlagen und ihrem Land aufgezwungen hat, der dazu führte, Grossprojekte einzukaufen und zu importieren und das Land zu verschulden. Und diese kritische Sicht ist durchaus angemessen. Es ist allerdings nicht unbekannt, dass sehr viele Entwicklungsorganisationen und andere Kreise, gerade während der 70er Jahre immer wieder auf Menschenrechtsverletzungen in solchen Staaten hingewiesen haben. Und auf die Gefahr hin, dass es nicht zusammengehört, möchte ich doch zeigen, worin ich den Zusammenhang erblicke : Wachsende Menschenrechtsverletzungen sind nämlich ein Gradmesser dafür, dass die Instabilität eines solchen Landes wächst. Und diese Instabilität haben Dritte-Welt-Organisationen im Falle Lateinamerikas und anderer Länder schon in den 70er Jahren deutlich aufgezeigt. Vielleicht hätte es guter Politik des geschäftlichen Interesses entsprochen, noch etwas mehr auf diese Instabilität zu achten.

IUED - Darf ich nochmals nachfragen : Wir wurden denn in den 70er Jahren die einzelnen Länderrisiken eingeschätzt, welche Kriterien wandte man an, um zu sagen : Für Exporte in dieses Land können wir voraussichtlich eine ERG-Garantie gewähren, oder welches Land erfüllt die Bedingungen nicht?

Hutzli - Die Kriterien sind im Grund die gleichen wie heute, doch sind sie verfeinert. Man verfügt heute über längerfristige Zahlen und besitzt auch zusätzliche Quellen, namentlich die internationalen Organisationen. Ein Faktor, der aber einfach nicht in Rechnung gestellt werden kann, ist die politische Entwicklung. Zahlreiche Länder kommen erst durch politische Ereignisse in wirtschaftliche Schwierigkeiten. Dies wird auch in Zukunft der Fall sein. Auch wenn alle wirtschaftlichen Faktoren auf eine günstige Entwicklung hindeuten, kann ein politischer Umschwung den wirtschaftlichen Aufbau zunichte machen.

Zu den Menschenrechten: In Afrika, wo die Menschenrechtsverletzungen häufig sind, ist das Engagement der ERG äusserst gering; zahlreiche afrikanische Länder sind schon seit langem von der ERG ausgeschlossen. Dies gilt ganz besonders für Länder, in denen Exportkredite fehl am Platze sind, weil sie die finanziellen Möglichkeiten des Landes übersteigen, und nur über Entwicklungs- und Finanzhilfe etwas geleistet werden kann. 
Nicht vergessen darf man auch, dass die ERG viele Gesuche ablehnt. Dies kommt natürlich in keiner Statistik zum Ausdruck.

Gerster - Es ist schon vor einiger Zeit das Stichwort "Finanzierungskonkurrenz" gefallen. Auf dieser Ebene entscheidet sich die Vergabe von Aufträgen vielfach. Das ist eine Realität, wie ich den Berichten immer wieder entnehmen muss, und da scheint mir ein sehr gefährliches Moment für risikobehaftete Geschäfte dabeizusein. Denn man kommt ja an der Tatsache nicht vorbei, dass die Kunden arme, finanzschwache Länder sind. Ich denke da jetzt nicht nur an Afrika, sondern durchaus auch an verschiedenen Länder mit mittlerem Einkommen, die sich diese Einfuhren nur auf Kreditbasis leisten können. Und in so und so vielen Fällen ist ja die Rückzahlungskapazität durch die mit den Darlehen finanzierten Projekte nicht gestiegen. Von daher sind Verluste vorprogrammiert, wenn man nicht wirklich sehr wählerisch ist.

Ich habe zur Kenntnis genommen, dass in den 70er Jahren eine restriktive Praxis der Fall gewesen ist. Ich kann das nicht beurteilen, ich finde es aber im Hinblick auf die Zukunft wichtig, dass die Zielsetzung der ERG nicht jene ist, um jeden Preis gewisse Märkte zu behalten oder sogar noch neue hinzuzuerwerben, wenn eben von der wirtschaftlichen Substanz der potentiellen Kunden her die Projekte längerfristig gar nicht bezahlt werden können. Ich glaube, das ist eine Realität, mit der die Exportindustrie leben muss, sofern sie nur mit minimalen Staatskrücken auskommen will.

Hutzli - Es ist bestimmt nicht so, dass die Exportindustrie jeden Auftrag "à-tout-prix" will. Wo die Risiken zu gross sind, geht die Firma selbst ein grosses Wagnis ein. Bei einem Garantiesatz von $70 \%$ trägt sie z.B. $30 \%$ des Risikos selbst. Die Interessen der Firma und der ERG decken sich damit.

Die Risiken bestehen aber nicht nur in armen Ländern. Wie schon erwähnt, können auch in an sich reichen Ländern durch politische Entwicklungen wirtschaftliche Schwierigkeiten entstehen. Wenn man jedes Land, in dem ein entferntes politisches Risikos besteht, ausschliessen wollte, könnte nicht mehr in viele Länder exportiert werden. Es ist aber gerade Aufgabe der ERG, die unberechenbaren politischen Risiken abzusichern.

Saladin - Wir müssen schon schauen, was die ERG ist : Sie ist eine Risikogarantie! Und Risiken führen hie und da zu Verlusten; es gibt keine Versicherung, die keine Schäden aufweist. Zum Vorschlag, restriktiv zu sein und nicht "à-tout-prix" Märkte zu erobern : Irgendwo geistert das Bild herum, als reise die schweizerische Exportindustrie in der Welt herum und würde "à-toutprix" jeden Auftrag hereinnehmen. Das ist ein total falsches Bild. Von zehn Möglichkeiten wird vielleicht eine realisiert. Ich meine, da herrscht ein harter Konkurrenzkampf. Am Schluss entscheidet nicht die ERG über das Projekt, sondern der Kunde und das Land. Auf die Gestaltung des Projektes hat die ERG praktisch keinen Einfluss. Es wird in der Regel durchgeführt oder nicht, 
mit oder ohne uns. Sie kann lediglich das Projekt ablehnen, wenn das Risiko allzu gross ist. Die Forderung nach besseren Projekten richtet sich in erster Linie an die Abnehmerländer selber; sie müssen in erster Linie eine vernünftige Politik treiben. Da können Weltbank, die regionalen Entwicklungsbanken und der IWF mithelfen. Damit möchte ich die Verantwortung der Exporteure für eine sorgfältige Projektbeurteilung und -gestaltung nicht herabmindern. Mitentscheiden bei der Projektgestaltung können wir im Rahmen der Entwicklungszusammenarbeit. Die Verwaltung kann hier mit dem Entwicklungsland verhandeln. Da muss man einfach die Unterschiede sehen, die bei den einzelnen Instrumenten der Zusammenarbeit mit den Entwicklungsländern bestehen. Die ERG ist kein Instrument der Entwicklungszusammenarbeit.

Gerster - Ich habe schon angetönt, wie unbefriedigend die Erfahrungen sind. Mit der Umschreibung der ärmeren Entwicklungsländer habe ich das bereits skizziert. Ich möchte gerade anhand dieses Stichworts darauf hinweisen, dass alle Formen der Entwicklungszusammenarbeit dem Bundesgesetz über internationale Entwicklungszusammenarbeit und humanitäre Hilfe unterstehen und von Gesetzes wegen schwergewichtig auf die ärmeren Entwicklungsländer ausgerichtet sind.

Bei den wirtschafts- und handelspolitischen Massnahmen werden als Obergrenze zur Definition der ärmeren Entwicklungsländer 1400 Dollar Pro-KopfEinkommen angewandt. Es wurde vor 2 Jahren ausdrücklich bekräftigt, dass dieses Bundesgesetz und dessen Ausrichtung auf die ärmeren Entwicklungsländer auch für die Mischkredite Geltung haben. Somit ergibt sich die Forderung nach einheitlicher Anwendung dieses Begriffes sowohl bei der ERG wie bei der Entwicklungszusammenarbeit ganz zwangsläufig. Es wäre verfehlt, zu verlangen, die Mischkredite auf die ärmsten Länder unter 600 Dollar auszurichten. Vielmehr ist es sinnvoll, die Obergrenze bei der ERG in Uebereinstimmung mit dem Entwicklungszusammenarbeitsgesetz ebenfalls bei 1400 Dollar zu ziehen.

Verschiedentlich hat es zwischen dem BAWI und den privaten Entwicklungsorganisationen Gespräche über einen möglichen Einbezug unserer Seite in das ERG-Entscheideverfahren gegeben. Diese Gespräche haben in der Substanz zu nichts geführt und sind wieder sistiert worden.

Dann die Frage der Grossprojekte : Wir meinen, das ist eigentlich ein Sonderproblem, für das sich auch ein gesondertes Verfahren rechtfertigen würde. Zum Teil richten sich unsere Vorstellungen bereits an den Gesetzgeber, nicht nur an die Verwaltung. Es liegt hier beispielsweise der Vorschlag eines Beschwerderechts der Entwicklungsorganisationen analog zur Umweltschutzgesetzgebung auf dem Tisch oder eines verbindlichen Konsultationsmechanismus innerhalb der Bundesverwaltung. 


\section{Das heutige Entscheidverfahren}

Saladin - Wenn Garantiegesuche kommen für Exporte in die 67 ärmeren Länder, die gewisse Grössenordnungen übersteigen, nämlich 5 Millionen bei Investitionsgütern, 3 Millionen für Konsumgüter, 2 Millionen für Dienstleistungen und 10 Millionen für Bauleistungen, dann geht das Gesuch u.a. an den Entwicklungsdienst des BAWI, der die entwicklungspolitische Prüfung vornimmt, aufgrund eigener Beurteilungen, aufgrund von Berichten der internationalen Finanzierungsinstitute und, wenn nötig, nach Rücksprache mit Experten in oder ausserhalb der Verwaltung. Ich sehe keine Schwierigkeit, dass in diesem Verfahren unsere Mitarbeiter einmal einen Experten der Hilfswerke beiziehen, wenn sie wissen, dass er im fraglichen Land ein Projekt bearbeitet, bei dem ein Zusammenhang mit einer zu garantierenden Lieferung besteht, oder dass die Hilfswerke für ein bestimmtes Land über ganz besondere Kenntnisse verfügen, die wir in der Verwaltung nicht besitzen.

Der Entwicklungsdienst ist auch beauftragt, mit der DEH Kontakte aufzunehmen, insbesondere dort, wo die DEH eine besondere Expertise aufweist und wo Schwerpunktländer betroffen sind. Wenn in einem Projekt wirklich prominent Umweltschutzfragen hervortreten würden, welche die Risikobeurteilung beeinflussen, könnten wir auch mit dem Bundesamt für Umweltschutz Kontakt aufnehmen.

Dass wir kleine Lieferungen jedesmal nach allen Aspekten untersuchen, ist nicht möglich, weil vom Entwicklungseffekt her gar keine Beurteilungsgrundlage vorliegen würde. Der Entwicklungsdienst gibt seine Stellungnahme ungefiltert an die Kommission. Auch die DEH kann ihre Berichte ungefiltert an die Kommission weitergeben. Das gleiche gilt für das Bundesamt für Umweltschutz.

Die Kommission entscheidet dann aufgrund der Abwägung der verschiedenen Elemente, wobei ganz klar ist, dass die Entwicklungspolitik mitberücksichtigt wird, aber nicht de jure den entscheidenden Einfluss haben muss. Bei Gesuchen von grundsätzlicher Tragweite und solchen, denen aus anderen Gründen eine besondere Bedeutung zukommt, entscheidet gemäss Verordnung der Bundesrat. Dies geschah in letzter Zeit einige Male. Der Bundesrat ist selbstverständlich frei, alle Gesichtspunkte in seinen Entscheid miteinzubeziehen. Kein Vorberatungsverfahren wird verhindern, dass der Bundesrat die entwicklungspolitischen Ueberlegungen im einen Fall als entscheidend, im andern als weniger entscheidend betrachtet. Für wichtige und grundsätzliche Entscheide ist also der Bundesrat zuständig, und ich sehe nicht, wie dies geändert werden könnte oder sollte.

Selbstverständlich sind das Parlament und die parlamentarischen Kommissionen informiert. Sie haben Einsicht in alle Akten, wenn sie das verlangen. Eine parlamentarische Kommission ist frei, von der Kommission und von der Verwaltung Einsicht in alle Akten zu verlangen, die sie wünscht. Dies wird auch gemacht, und wir haben nichts zu verbergen. Das Parlament nimmt seine Aufsicht wahr. 
Zum Begriff der ärmeren Länder : Es gibt in der schweizerischen Entwicklungspolitik keine allgemein gültige Definition der ärmeren Entwicklungsländer, und zwar bewusst nicht, weil die Gruppe der ärmeren Länder in bezug auf die gesetzlichen Zielsetzungen ja für die einzelnen Instrumente immer wieder neu definiert werden muss! Bei der Nahrungsmittel- und Katastrophenhilfe z.B. spielt dieser Begriff keine grosse Rolle; bei der technischen Hilfe hat die BSP-pro-Kopf-Grenze eine andere Bedeutung, als sie für die Mischkredite hat. Für Mischkredite ist ja die Pro-Kopf-Grenze eine obere Limite : Wir schliessen die am weitesten fortgeschrittenen Länder aus. Wir schliessen aber auch die ärmsten, die "least-developed countries" aus. Wir wollen also mit Mischkrediten eine mittlere Kategorie der Entwicklungsländer erreichen, welche solche Kredite administrativ und wirtschaftlich absorbieren können. Mit der technischen Hilfe z.B. wollen wir gerade jene Länder erreichen, die administrativ und wirtschaftlich schwächer sind als die Länder der mittleren Kategorie.

Bei der ERG ist die Sache wiederum anders. Wir wollen vermeiden, dass Zielkonflikte mit der öffentlichen Entwicklungszusammenarbeit des Burndes entstehen. Und die entsprechenden Länder berücksichtigen wir in der ERG. Ich möchte, dass man in der Diskussion diese differenzierte Betrachtungsweise wirklich auch berücksichtigt.

Sie haben weiter gesagt, die Gespräche zwischen uns und Ihnen seien sistiert worden. Wenn ich mich recht erinnere, sind Sie von Ihrer Seite sistiert worden.

Das Gespräch zwischen BAWI und Hilfswerken kann durchaus noch vertieft werden, z.B. in dem Sinne, dass Sie uns Informationen über Länder liefern, wo Sie Experten im Einsatz haben, über welche die DEH und das BAWI nicht verfügen. Wir greifen gerne auf aussenstehende Experten zurück. Aber dass Sie ins Entscheidverfahren, das ja in der Verordnung festliegt, direkt eingreifen könnten, soweit können wir bei der heutigen Rechtslage nicht gehen.

Hutzli - Der Anteil der ärmeren Länder am Engagement der ERG ist sehr gering. In der Pressemitteilung ist der Prozentsatz genannt. Er ist gegenüber $14 \%$ im Jahre 1983 auf $17 \%$ im Jahre 1984 gestiegen; der Grossteil entfällt auf 4 Staaten. Die ganz armen Länder sind ohnehin gesperrt und kommen für Grossprojekte nicht in Frage. Die Bedeutung dieser wirklich ärmsten Länder darf deshalb nicht überschätzt werden.

Bei grösseren Projekten gibt jeweils auch die Schweizerische Botschaft im betreffenden Lande eine Stellungnahme ab, weshalb der Bundesrat in Kenntnis aller Gesichtspunkte urteilen kann.

Zu Manantali noch ein Hinweis : Als die ERG gewährt wurde, war das Projekt bereits im Bau. Der Entscheid der Schweiz hatte auf die Verwirklichung des Staudamms überhaupt keinen Einfluss mehr. 
Gerster - Um am letzten Punkt anzuschliessen : Manantali als Sonderfall ? Ich nehme zur Kenntnis, dass es wenige derart grosse Projekte gibt. Im übrigen kann ich das wiederum nicht beurteilen, weil es auch bei Grossprojekten keine Transparenz im Sinne einer klaren Liste ERG-gedeckter Grossrisiken gibt.

Und hier liegt der Hase im Pfeffer, Herr Saladin, wenn Sie sagen, Beiträge an die Verwaltung könnten immer eingebracht werden! Das wissen wir. Aber wir wissen ja nicht, zu welchen Projekten! Denn wir haben keine Angaben, für welche Projekte überhaupt ERG-Gesuche hängig sind. Ich nehme auch gerne zur Kenntnis, dass das BAWI die Hilfswerke um eine Stellungnahme bitten kann. Es ist allerdings so, dass seit $11 / 2$ bis zwei Jahren eine detaillierte Liste bei Ihnen liegt, in der 7 grosse Hilfswerke angegeben, in welchen Ländern sie tätig sind und wo sie allenfalls Informationen beschaffen könnten. Seither ist keine einzige Anfrage gekommen. Deswegen habe ich das Wort sistiert gebraucht. Aufgrund unserer Korrespondenz mit dem BAWI und den Angaben, die wir Ihnen über unsere Tätigkeit in den Entwicklungsländern gemacht haben, hat ein Gespräch stattgefunden. Das Resultat bestand in der Erkenntnis, dass die Zusammenarbeit schwierig ist und im Moment keine Möglichkeiten vorhanden sind, die Zusammenarbeit zu aktivieren. Ich weiss deshalb nach dem Resultat dieses Gesprächs nicht, mit welchen Vorschlägen wir noch an Sie gelangen sollen. Meines Erachtens sieht die Situation von aussen eben ganz anders aus, als Sie die Lage von innen her wahrnehmen.

Nun noch zum Punkt der ärmeren Entwicklungs/änder. Ich glaube, der Begriff hat sowohl bei der ERG als auch der Entwicklungszusammerarbeit eine ähnliche Funktion. Im Falle der Mischkredite dient der Begriff dazu, eine Obergrenze festzulegen, nach unten ergibt sich die Abgrenzung aus dem Absorptionsvermögen der Entwicklungsländer für Schweizer Erzeugnisse und aus ihren finanziellen Möglichkeiten.

Saladin - Wie bereits erwähnt, ist das BAWI bereit, die Gespräche weiterzuführen. In der Differenzierung der Anwendung der einzelnen Instrumente unserer Beziehungen zu den Entwicklungsländern, je nach ihrem Entwicklungsstand, liegt eine bewusste Politik

Gerster - Ja, gut - aber darin spiegelt sich wiederum die Realität in den Entwicklungsländern. Hingegen könnte man sehr wohl an reichere Länder Mischkredite vergeben. An und für sich wäre das machbar, aber dann stösst man an die Obergrenzen. Als vor 2 Jahren die Mischkredite im Rahmen der Arbeitsbeschaffungsmassnahmen um 100 Mio aufgestockt worden sind, da haben wir von den Hilfswerken her diesen Entscheid insofern kritisiert, als einmal mehr die besser gestellten Entwicklungsländer mehr Mittel erhalten. Weil wir damals im Rahmen der Petition "Entwicklungshilfe ist eine Ueberlebensfrage" um eine Ausdehnung der gesamten öffentlichen Entwicklungshilfe gekämpft haben, forderten die Hilfswerke, man müsse nicht nur an 
die reicheren Entwicklungsländer mehr Mischkredite vergeben, sondern unbedingt auch die Leistungen für die ärmeren Entwicklungsländer verstärken. Recht bald nach dieser Verlautbarung von unserer Seite wurden zu den Mischkrediten neue Richtlinien verabschiedet. Und diese Richtlinien wurden vom Bund her damit gerechtfertigt, dass auch die Mischkredite dem Entwicklungszusammenarbeitsgesetz unterstehen und sich ebenfalls an ärmere Entwicklungsländer richten. Der Begriff ärmere Entwicklungsländer wurde also ausdrücklich auch auf die Mischkredite angewandt.

\section{Schuldenkonsolidierungen führen zu vorsichtiger Garantiegewährung}

IUED - Der nächste Diskussionspunkt betrifft die Bedeutung der Schuldenkonsolidierung für die ERG, insbesondere für ihre Eigenwirtschaftlichkeit. Schuldenkonsolidierungen haben sich wegen vermehrter Zahlungsbilanzprobleme einzelner Entwicklungs/änder erhöht, und die Schweiz ist am Abschluss solcher Konsolidierungen mitbeteiligt. Welche Bedeutung haben diese bei einer allfälligen Gewährung neuer Garantien und für die Wiederherstellung der Eigenwirtschaftlichkeit der ERG?

Saladin - Zuerst zum Tatbestand. Wir haben heute 630 Millionen Franken Konsolidierungsguthaben. Das sind im Prinzip Guthaben in der Bilanz der ERG. Denen stehen die 600 Millionen Vorschüsse des Bundes gegenüber. Die Bewertung der Konsolidierungsguthaben wirft natürlich viele Fragen auf. In der Regel haben bisher eigentlich alle Länder ihre Verpflichtungen im Rahmen von Konsolidierungen erfüllt. Es gibt Rekonsolidierungen; praktisch alle Länder erkennen aber ihre Schulden an und sind bereit, diese zurückzuzahlen. Wertberichtungen bei diesen Konsolidierungsguthaben könnten wohl nur im Rahmen international abgestimmter Aktionen erfolgen.

Es gibt verschiedene Gründe für Konsolidierungen. Die einen Länder geraten in eine momentane Zahlungskrise, weil sie sich übernommen haben. Andere kennen strukturelle, langfristige Zahlungsprobleme. Diese Fälle sind in bezug auf die ERG-Politik genau auseinanderzuhalten.

Eine Schuldenkonsolidierung findet ja meistens nur statt, wenn das Land mit dem Währungsfonds ein Strukturanpassungsprogramm vereinbart hat. Diese Programme sind unterschiedlich gestaltet. Auch wenn viele Länder ihre Importe beschränken müssen, ist es doch wichtig, dass die wesentliche Importe tätigen können, damit die Produktionskapazität ausgenützt wird.

In der Vorphase einer Konsolidierung will man zuerst wissen, wie verhält sich das Land, welche Programme werden gemacht, und da erteilt die ERG praktisch keine Garantie. Wenn das Programm steht, muss man abklären, welche Garantiepolitik sich in bezug auf ein bestimmtes Land aufdrängt.

Schöb - ERG und Banken stehen vor den gleichen Problemen, die ERG setzt sich im Rahmen des Pariser Klubs, die Banken bei den Schuldenrestrukturie- 
rungen der nicht-ERG-gedeckten Kredite mit weitgehend gleichen Schwierigkeiten auseinander. Die Banken haben ein grosses Interesse daran, Schuldner zu haben, die ihre Schulden bedienen können, die ihre vertraglichen Verpflichtungen einhalten. Wenn dies eine Umstrukturierung der Schulden bedingt, so stehen die Banken dazu und tragen das ihre bei. Bis eine solche Restrukturierung steht und implementiert ist, kann es leider Monate, sogar Jahre dauern. In dieser Zwischenphase haben wir Schwierigkeiten, eine Kreditpolitik zu formulieren. Wir müssen oft gewisse Kreditlinien aufrechterhalten, damit lebensnotwendige Importe abgewickelt werden können. Ein echter Schritt vorwärts in Form einer neuen, vorsichtig offenen Kreditpolitik kann aber erst gemacht werden, wenn ein allseitig akzeptables Wirtschaftsprogramm unter Dach ist, das das Vertrauen des Auslandes, insbesondere der Geldgeber geniesst.

Saladin - Die neuesten Weltbankberichte betonen, welch wichtige Rolle Exportkredite heute für viele Länder bei der Bewältigung dieser schwierigen Uebergangsphase spielen können. Die Frage, welche Rolle den Exportkrediten im Anpassungsprozess zukommt, muss anhand der entsprechenden Programme der einzelnen Länder beurteilt werden.

Rambousek - Wir haben von der Bank aus berechnet, dass es in den nächsten beiden Jahren in den gefährdeten Ländern $\mathbf{4 0}$ Milliarden US-Dollar "new money" braucht. Ein grosser Teil davon kann durch eine verstärkte und konstante Handelsfinanzierung geleistet werden. Das ist ein wichtiger Punkt, mit dem wir einen Beitrag dazu leisten können, die Produktionsstruktur dort zu modernisieren und zu verändern, wo dies für den wirtschaftlichen Anpassungsprozess wichtig ist.

Schöb - Wir sehen bei unserer Bank die Entwicklung längerfristig so, dass Weltbankkredite, IFC- und IDB-Kredite und Exportfinanzierungen für grosse Projekte bereitgestellt werden müssen, um den langfristigen Anpassungsprozess in den hochverschuldeten Ländern erfolgreich durchziehen zu können. Projekte, die die Unterstützung dieser internationalen Organisationen geniessen, können teilweise auch mit Krediten finanziert werden, die die Banken aufgrund kommerzieller Ueberlegungen bereitstellen. Die Finanzierung muss mit diesem Schwergewicht geschnürt werden, und die Exportkredite sind daraus nicht wegzudenken.

Saladin - Die Schwierigkeit für das Entwicklungsland besteht darin, festzulegen, was prioritär ist. Die staatlichen Entscheidstrukturen sind sehr unterschiedlich. Bei den einen hat der Staat viel zu sagen, bei den andern weniger. Wir müssen aber auf von den Ländern erstellten Programmen abstellen. Wichtig ist, dass wir die Funktion der Kredite und die Verantwortung sehen, die wir als Schweiz und als ERG in diesem schwierigen Prozess innehaben. 
Kappeler - Wenn schon konsolidiert werden muss und die Schulden überhand genommen haben, dann verlagert sich die entwicklungspolitische Bedeutung der öffentlichen Haltung in der Schweiz. Nicht nur die Frage der ERG und der weiteren Gewährung von Handelskrediten, sondern auch das Verhalten der Schweiz als Gläubiger ist bei diesen Konsolidierungsfragen sehr wichtig.

Die Berücksichtigung von Art. 5 durch die schweizerischen Unterhändler, wie es auch unsere und entwicklungspolitische Kreise gefordert haben, gewinnt in dieser Phase an Bedeutung. Ich glaube, das ist viel wichtiger als die wahrscheinlich monatelang eingefrorenen neuen Kredite.

Saladin - Hier müssen Sie sich der Stellung der Schweiz und unserer Gewichtes bewusst sein. Wir stehen in den meisten Konsolidierungsländern im Verhältnis zu anderen Staaten mit kleineren Beträgen zu Buche. Sie häufen sich aber jetzt zu 630 Millionen Franken zusammen. Als einzelnes Land haben wir relativ wenig Spielraum für generösere Lösungen. $\mathrm{Zu}$ bedenken ist auch, dass der Bund immer über Forderungen verhandelt, die teilweise - im Umfang des Selbstbehaltes - nicht ihm, sondern dem Exporteur zustehen.

Gerster - Vorher wurden die Anpassungsprogramme angesprochen. Bei diesen haben die wirtschaftspolitischen Prioritäten entscheidende Folge. Sie wissen, dass gerade in Afrika die Anpassungsprogramme des Währungsfonds - soweit sie in den letzten Jahren realisiert worden sind - eine gehörige Rate an Misserfolgen aufzuweisen haben. Dies mag in Einzelfällen am schlechten Willen der Beteiligten auf der afrikanischen Seite gelegen haben, aber es liegt ohne Zweifel auch in einer ungenügenden Wahrnehmung der sozialen und politischen Realitäten dieser Länder seitens des IWF. Die Rezepte werden zu sehr von aussen her eingebracht. Darauf deuten auch die Unterschiede von weltbankfinanzierten Anpassungsprogrammen und IWF-Programmen. Ich möchte in diesem Sinne die Bedeutung "angepasster" Anpassungsprogramme unterstreichen.

Ist eine erweiterte Transparenz politisch möglich ?

IUED - Wir müssen zur letzten spezifischen Frage kommen, zur Transparenz der ERG : In der Diskussion um die ERG fällt aus entwicklungspolitischen und wissenschaftlichen Gründen die Forderung nach vermehrter Transparenz. Welches sind die Gründe der bisherigen summarischen Informationspolitik ? Ueber welche Aspekte der ERG wäre Ihrer Meinung nach eine vertiefte Information nötig, und in welchem Rahmen wäre dies möglich ?

Kappeler - Es gäbe drei Kanäle der Transparenzschaffung. Der eine ist gegeben durch die Verantwortung der Bundesbehörden gegenüber dem Parlament, insbesondere gegenüber den Kommissionen des National- und des Ständerates. Die eidgenössischen Räte haben ein generelles Aufsichsrecht.

Ein zweiter Kanal wäre der spezifisch schweizerische Weg der Mitbeteiligung verschiedener wirtschaftlicher Kreise an der Durchführung staatlicher Programme. Dies geschieht meistens durch Einsitznahme in begleitenden Gremien. 
Und drittens die direkte Information der Oeffentlichkeit durch Presse und Medien.

Oeffentlichkeit und Parlament lasse ich jetzt einmal beseite. Ich möchte mich auf die 2. Stufe konzentrieren. Aufgrund ihrer finanzpolitischen, ihrer entwicklungspolitischen und ihrer strukturpolitischen Bedeutung ist die ERGKommission ungenügend zusammengesetzt, sogar in einer für die Schweiz höchst unüblich restriktiven Art. Sonst sind alle Gremien sämtlicher Departemente aus den massgeblicheren Kreisen des Landes zusammengesetzt. Hier stellen wir fest, dass dem nicht so ist. Und wir stellen sogar fest, dass, wie auch in dieser Diskussion, immer das Arbeitsplatzargument zuvorderst als Begründung der ERG hingestellt wurde, dass aber die Gewerkschaften empörenderweise in diesem Gremium fehlen. Wir lassen uns mit dieser Situation auf die Dauer nicht befriedigen.

Gerster - Die Gründe, die für eine vermehrte Mitbeteiligung der privaten Hilfswerke an der ERG sprechen, sind im Verlauf unseres Gesprächs verschiedentlich offensichtlich geworden. Sie liegen im entwicklungspolitischen Bereich, in der Notwendigkeit einer Uebereinstimmung Entwicklungspolitik Exportförderung. Sie liegen überdies auch im Bereich der Transparenz. Wenn die Engagements bekannt sind, welche die ERG in den einzelnen ländern eingegangen sit, können diese Risiken von einer breiteren Oeffentlichkeit eingeschätzt werden. Das ist ein alter Wunsch von unserer Seite, der immer mit Hinweis auf diplomatische Komplikationen zurückgewiesen worden ist. Darüber würde ich mich gerne noch etwas näher unterhalten, weil diese Begründung einfach nicht stichhaltig ist.

Dann hat Herr Kappeler völlig zu Recht auf die strukturpolitischen Fragen hingewiesen, die durch die ERG aufgeworfen werden, und diese als zusätzlichen Grund vermehrter Transparenz angeführt.

Saladin - Das Parlament kann über sämtliche Informationen verfügen, die es wünscht. Dem steht nichts entgegen. Und wir geben dem Parlament offen Auskunft.

Ich möchte festhalten, dass die Kommission ein operationelles Organ ist, was Einfluss auf die Zahl der Kommissionsmitglieder hat. Die Kommission verabschiedet 50, 60, 70 Fälle pro Sitzung zuhanden der gemäss Verordnung zuständigen Entscheidorgane. Die Zusammensetzung der Kommission ist ein politischer Entscheid. Sie kennen die bisherigen Stellungnahmen des Bundesrates; die Stellungnahme zur Motion Renschler wird neu hinzukommen.

Oeffentlichkeitsinformation : Ich glaube, dass wir in der Informationspolitik noch nicht das Maximum dessen erreicht haben, was sich machen liesse. Eine Grenze bildet das Geschäftsgeheimnis der Unternehmer. Dessen Schutz müssen wir ernst nehmen. Dann gibt es Grenzen in bezug auf die Einschätzung von Länderrisiken und deren Veröffentlichung. Deshalb geben wir, wie alle ERG-Institute, keine Zahlen betreffend die Garantiesätze oder Länderpla- 
fonds bekannt. Man würde damit Signale setzen, die trotz Streben nach Objektivität doch immer subjektiv bleiben. Zudem könnten die Beziehungen zu einzelnen Ländern belastet werden.

Wir streben eine möglichst sachliche Durchführung der ERG-Politik an, die dem Exporteur wirklich etwas bringt. Wir wollen eine "Verpolitisierung" der ERG vermeiden.

Kappeler - Die ganze Diskussion zeigt doch seit Jahren, dass das einfach kein apolitisches Gebiet ist. Es gibt doch immer Einschätzungen, Werturteile, Einstufungen, und die verschiedenen Gesichtspunkte sollte man integrieren.

Gerster - Wir haben gewünscht, dass am Jahresende die länderweisen Garantiesummen publiziert werden, nicht die Garantiesätze. Garantiesätze signalisieren bis zu einem gewissen Grad die Kreditwürdigkeit. Die Bekanntgabe der Länderplafonds haben wir auch nie gewünscht. Zu Beginn war es eine ganz simple Sache, zu wünschen, dass man über die Verschuldung oder das ERGEngagement in gewissen Ländern informiert wird. Es hat sich dann herausgestellt, dass das nicht möglich sein soll. Und die Begründung dafür hat nie eingeleuchtet. Ich möchte die Gegenfrage stellen, ob es einen Unterschied signalisiert, wenn in Aegypten 500 Mio, 1 Mia Franken, 2 Mia Franken Garantie ausstehend sind. Das hat mit der Kreditwürdigkeit von Aegypten nichts zu tun. Es ist ein ganz allgemeiner Wunsch nach mehr Information über die Beziehungen zwischen der Schweiz und den Entwicklungsländern. Ich möchte Sie darauf hinweisen, dass z.B. Exportkredite und -garantien der staatlichen amerikanischen EXIM-Bank nach Ländern geordnet im Detail nachzulesen sind.

\section{Wird die ERG mit dem Welthandel wachsen ?}

IUED - Wir möchten zur letzten Frage unserer Diskussion kommen. Wenn man längerfristig an die zukünftigen Handelsbeziehungen der Schweiz mit den ärmeren Entwicklungs/ändern und den Schwellenländern denkt, wie werden diese gestaltet sein ? Welche Rolle spielt dabei die ERG ? Und wie schätzen Sie im Rahmen dieser Beziehungen die Möglichkeit einer verstärkten Eigenwirtschaftlichkeit der ERG ein?

Rambousek - Ich möchte diese Frage kurz beantworten. Ich kann mir eine multipolare Welt vorstellen, im Gegensatz zu einer eher bipolaren von heute, viele gleichwertige Zentren also, eine Arbeitsteilung, die verfeinerter und internationaler ist als heute, aber das ist meine persönliche Meinung. In Szenarien, wie sie heute diskutiert und präsentiert werden, noch die genaue Position einer künftigen ERG zu bestimmen, scheint mir nicht möglich.

Kappeler - Ich würde zusammenfassend sagen, das Instrument der ERG ist nicht einfach schlecht und muss über Bord geworfen werden. Ich würde 
meinen, mit mehr Transparenz, mit einer konsequenten Beachtung entwicklungspolitischer Fragen bei den Einzelentscheiden, kann man das Instrument belassen. Ich würde nebst diesen zwei Erfordernissen sagen, es braucht in der Wirtschaftspolitik der Schweiz das Bewusstsein, dass dieses Instrument ein Element der Strukturpolitik ist, dass man die anderen Elemente dazu in Beziehung setzen soll, und dass man die anderen Instrumente der Strukturpolitik vielleicht etwas "larger" ansehen soll. Arbeitsplatzschaffung und Arbeitsplatzerhaltung kann auch mit anderen als mit diesem bis jetzt relativ teuren Instrument betrieben werden. Ich lenke also den Blick eher von der ERG weg und sage, wenn das Instrument im weiteren Rahmen gesehen wird und mit Transparenz und unter Beachtung von Art. 5 gehandhabt wird, dann sehe ich für die ERG selbst keine umstürzenden Veränderungen und Veränderungszwänge voraus.

Gerster - Ich habe schon früher darauf hingewiesen, dass mittelfristig und längerfristig ein schrumpfendes Volumen an Absatzmöglichkeiten in der Dritten Welt vorhanden sein wird. Unter Dritter Welt meine ich nicht nur die ärmeren Länder, sondern auch die Schwellenländer in Lateinamerika. Unsere Politik müsste darauf ausgerichtet werden, einen derartigen Wandel nicht durch eine zu grosszügige Kreditgewährung zu verhindern, sondern den Wandel vorwegzunehmen und zu akzeptieren. Möglicherweise wird dies schwer realisierbar sein, weil es auf unserer Seite vermutlich in wachsendem Masse Ueberkapazitäten in bestimmten Bereichen geben wird. Und die Gefahr besteht, derartige Ueberkapazitäten auch durch Exporte nach Entwicklungsländern abzubauen, wie das jetzt beispielsweise mit der Umwandlung von Agrarüberschüssen in Nahrungsmittelhilfe geschieht. Ich meine auch, dass verantwortliches Handeln in der Privatwirtschaft beinhaltet, beim Wettlauf um fragwürdige Projekte nicht mitzumachen.

Schöb - Ich möchte weniger auf Zukunftsperspektiven eingehen, als auf das, was aus der Sicht der Banken für die ERG und die Exportindustrie richtig wäre. Unser Anliegen ist, dass wir die Stärken der Exportindustrie und der damit verbundenen Dienstleistungen der Banken zum Tragen bringen, und nicht durch unflexible Instrumente behindert werden. Die Spiesse der Schweizer Exporteure sollen nicht noch kürzer werden, sie sind im Vergleich mit den Instrumenten der wichtigsten Industriestaaten jetzt schon kurz.

IUED - Wir möchten die Frage nach der Eigenwirtschaftlichkeit der ERG noch einmal stellen. Kann die ERG in einer näheren Zukunft wieder eigenwirtschaftlich funktionieren?

Hutzli - In der näheren Zukunft wird sich die Tendenz verstärken, dass die Industriestaaten und die Schwellenländer, die zu den Industriestaaten aufstiessen, unsere Hauptmärkte sein werden. Es gibt ja eine ganze Reihe, die nicht mehr weit zurückliegen. Andererseits befürchte ich, dass sich mit dem 
bisherigen System die ganz armen Länder einfach nicht mehr selbst retten können. Niemand hat ein Rezept, wie man gerade die afrikanischen Staaten wieder emporbringen kann, namentlich, wie man sie in das Welthandelssystem integrieren könnte.

Im Bereich der Eigenwirtschaftlichkeit der ERG sind in diesem Frühjahr ganz entscheidende Schritte gemacht worden. Durch die Sistierung der Währungsabsicherung ist eine grosse Verlustquelle gestopft worden. Und die rund 50 \%ige Gebührenerhöhung wirkt einschränkend. Ob sich diese Erhöhung auf die Rechnung positiv oder negativ auswirken wird, kann noch nicht gesagt werden. Die Exporteure können auch noch zurückhaltender, noch selektiver vorgehen, wenn es darum geht, ein Geschäft der ERG zu unterstellen oder nicht. Die Resultate dieser Verteuerung - es ist ja nicht die erste in den letzten Jahren - zeigten sich aber schon im markanten Rückgang des ERGEngagements. Vor 1979 befand es sich auf $25 \mathrm{Mia}$ und jetzt ist es auf $15 \mathrm{Mia}$ zurückgefallen. Anderseits muss man auch sagen, dass es : zuerst von $3 \mathrm{Mia}$ anfangs der 70er Jahre auf 25 Mia geschnellt ist.

In relativ kurzen Zeiträumen werden Bewegungen ausgelöst, die eine Prognose erschweren. Die Selbsttragung wird ganz entscheidend von der wirtschaftlichen Situation der Schuldnerländer abhängen, davon, ob diese ihre Schuldenkrisen überwinden können und ihre Konsolidierungsverpflichtungen honorieren. Heute sind die Guthaben der ERG aus Konsolidierungen ungefähr gleich hoch wie die Verpflichtungen der ERG : rein rechnerisch ist sie damit noch gar nicht in den roten Zahlen. Die Garantien, die jetzt neu ausgegeben werden, dürften - eigentliche Katastrophen vorbehalten - eine Selbsttragung gewährleisten.

Saladin - Ich kann nur noch beifügen, dass wir den Zweck des Instrumentes, nämlich dem Exporteur in seinen Bemühungen um die Durchdringung der Märkte zu heifen, immer im Auge behalten müssen. Das ist das erste; und das zweite : Die ERG ist ein Instrument, das auf gesetzlicher Grundlage beruht, damit auch im politischen Bereich angesiedelt ist und deshalb auch Veränderungen erfahren kann, wie bereits die Vergangenheit gezeigt hat.

IUED - Wir möchten Ihnen sehr herzlich danken, dass Sie sich die Zeit genommen haben, an diesem Gespräch teilzunehmen. 


\section{EXPORTRISIKOGARANTIE (ERG)}

Die Exportrisikogarantie wurde 1934 zusammen mit anderen Massnahmen zur Krisenbekämpfung geschaffen. Sie ist eine Versicherung des Bundes für besondere, mit Ausfuhrgeschäften verbundene Risiken. Anfänglich war die Versicherungsdeckung sehr beschränkt. Im Lauf der Zeit wurde sie erweitert. Versicherbar sind Risiken aufgrund politisch und wirtschaftlich unsicherer Verhältnisse im Ausland. Die Absicherung gegen Verluste aus Wechselkursänderungen wurde auf den 1. April 1985 sistiert. Unter dem Begriff der politischen Risiken können die folgenden, vom Gesetz als versicherbare Schadensereignisse aufgezählten Tatbestände zusammengefasst werden : Transferschwierigkeiten, Moratorien, Zahlungsunfähigkeit oder Zahlungsverweigerung von Staaten und anderen öffentlichrechtlichen Körperschaften sowie von Betrieben des privaten Rechts, die öffentliche Aufgaben erfüllen, ausserordentliche ausländische staatliche Massnahmen oder politische Ereignisse im Ausland, welche privaten Schuldnern die Erfüllung verunmöglichen, zur Beschlagnahme oder Beschädigung von Waren des Exporteurs führen. Sofern der Exporteur einen entsprechenden Antrag stellt, ist auch die Unzumutbarkeit oder die Unmöglichkeit einer Lieferung infolge eines der aufgezählten Ereignisse versicherbar (sog. Fabrikationsrisiko).

Von Gesetzes wegen ausgeschlossen ist die Versicherung der Zahlungsunfähigkeit oder Zahlungsverweigerung privater Besteller (privates Delkredererisiko). Versichert werden können die Lieferung oder Vermietung von Waren, die Herstellung von Werken, die Leistung von Bau-, Ingenieur- und Entwicklungsarbeiten, die wissenschaftliche, technische und wirtschaftliche Beratung, sowie die Ueberlassung von Lizenzen und anderen immateriellen Güterrechten. Die Lieferungen und Leistungen müssen schweizerischen Ursprungs sein. Ausnahmen können gewährt werden für Zulieferungen oder einzelne Bestandteile, deren Beschaffung in der Schweiz nicht möglich oder nicht zumutbar ist, sowie für angemessene Lokalausgaben und ausländische Leistungen, die im Zusammenhang mit schweizerischen Lieferungen stehen. Garantienehmer können nur Firmen sein, die in der Schweiz niedergelassen und im Handelsregister eingetragen sind. Die Bemessung der Gebühr wird in der Verordnung geregelt. Die Gebühreneinnahmen sollen langfristig die Eigenwirtschaftlichkeit des Fonds für die ERG gewährleisten.

Die Verwaltung der ERG wird von einer Geschäftsstelle in Zürich, bei der die Gesuche einzureichen sind, geführt. Eine vom Bundesrat eingesetzte Kommission begutachtet die Gesuche und Entscheide über die Deckung von Verlusten. Der Entscheid über die Gewährung von Garantien liegt je nach der Höhe der Garantie beim Bundesamt für Aussenwirtschaft oder beim Eidg. Volkswirtschaftsdepartement. Ueber Gesuche, die von grundsätzlicher Tragweite sind, oder denen aus anderen Gründen eine besondere Bedeutung zukommt, entscheidet der Bundesrat.

Die gesetzliche Regelung war zuerst in befristeten Bundesbeschlüssen und schliesslich in einem Bundesgesetz vom 6. April 1939 enthalten. Dieses wurde durch das Bundesgesetz vom 26. September 1950 über die Exportrisikogarantie abgelöst. Seitherige Teilrevisionen betrafen die Aufhebung der Selbstkostenklausel (BG vom 21. März 1973), die Verringerung des Selbstbehalts durch die Erhöhung des maximal möglichen Garantiesatzes auf $95 \%$ des Lieferungsbetrages (BB vom 20. Juni 1975 und BG vom 23. Juni 1978), die Verselbständigung der ERG-Rechnung durch die Schaffung eines Fonds für die ERG und die Bestimmung, dass bei Exporten nach ärmeren Entwicklungsländern die Grundsätze der schweizerischen Entwicklungspolitik mitzuberücksichtigen sind (BG vom 1. Oktober 1980). Die Ausführungsbestimmungen sind in einer Verordnung enthalten.

Quelle : Eidg. Volkswirtschaftsdepartement, Presse- und Informationsdienst. 